\title{
Sociodemographic, behavioural and environmental correlates of sweetened beverage consumption among pre-school children
}

\author{
Roman Pabayo ${ }^{1,2}$, John C Spence ${ }^{1, *}$, Nicoleta Cutumisu' ${ }^{1}$ Linda Casey ${ }^{3}$ and \\ Kate Storey ${ }^{4}$ \\ ${ }^{1}$ Faculty of Physical Education and Recreation, University of Alberta, E-488 Van Vliet Centre, Edmonton, Alberta, \\ Canada, T6G 2H9: ${ }^{2}$ Department of Society, Human Development, and Health, Harvard School of Public Health, \\ Boston, MA, USA: ${ }^{3}$ Stollery Children's Hospital/Agricultural, Food and Nutritional Science, Edmonton, Alberta, \\ Canada: ${ }^{4}$ School of Public Health, University of Alberta, Edmonton, Alberta, Canada
}

Submitted 8 June 2011: Accepted 8 December 2011: First published online 24 January 2012

\begin{abstract}
Objective: To identify sociodemographic and environmental correlates of sweetened beverages (regular soft drinks, fruit juice) among children of pre-school age.

Design: Children's dietary intake, food behaviours and screen time were measured by parental report. A Geographic Informational System was used to assess the number of grocery stores and fast-food restaurants available within $1 \mathrm{~km}$ of the children's residence. Multivariate log-binomial regression models were constructed to determine correlates of drinking soft drinks during the previous week.

Setting: Edmonton region, Canada.

Subjects: Children aged 4 and 5 years ( $n$ 2114) attending a public health unit for immunization were recruited for a cohort study on determinants of childhood obesity, between 2005 and 2007.

Results: Children from neighbourhoods with low socio-economic status (relative risk $(\mathrm{RR})=1 \cdot 17,95 \% \mathrm{CI} 0 \cdot 98,1 \cdot 40)$ or who participated in $>2 \mathrm{~h}$ of screen time daily $(\mathrm{RR}=1 \cdot 28,95 \% \mathrm{CI} 1 \cdot 13,1 \cdot 45)$ were significantly more likely to have consumed regular soft drinks within the last week. Those who lived within $1 \mathrm{~km}$ of a grocery store were significantly less likely to consume regular soft drinks (RR $=0 \cdot 84,95 \% \mathrm{CI}$ $0.73,0.96$ ). Children who participated in $>2 \mathrm{~h}$ of screen time daily ( $\mathrm{RR}=1 \cdot 16$, $95 \%$ CI $1 \cdot 06,1 \cdot 27$ ) were more likely to exceed the recommended weekly number of servings of fruit juice.

Conclusions: Socio-economic and built environment factors are associated with soft drink consumption in children of pre-school age. These findings may help health professionals to advocate for policies that reduce soft drink consumption among children.
\end{abstract}

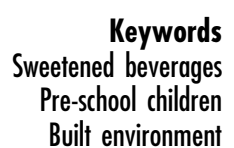

The proportion of Canadian children classified as being overweight or obese has risen from $14 \%$ to $31 \%$ among boys, and from $14 \%$ to $25 \%$ among girls, in the period between 1981 and $2007^{(1)}$. This trend is alarming because excess adipose tissue in children increases the risk of CVD and diabetes in adulthood ${ }^{(2)}$. Secular trends of increasing energy intake in recent years ${ }^{(3)}$ may underlie the rising prevalence of overweight and obesity among youth in developed nations ${ }^{(4)}$. Therefore, public health professionals and researchers need to identify factors that contribute to excess energy intake among children.

A likely source of excess energy intake, and thus a potential contributor to the increased prevalence of overweight and obesity, is the consumption of sweetened beverages such as regular soft drinks and fruit juices ${ }^{(5-7)}$. A regular soft drink is a non-alcoholic beverage that typically contains water, sugar and high-fructose corn syrup as opposed to a diet soft drink, which contains a non-caloric sugar substitute. Since the end of World War II, sugar intake has increased dramatically as a result of soft drinks becoming a dietary staple in most countries ${ }^{(5)}$. Recent evidence indicates regular soft drink consumption has been increasing among children in the USA and Europe $^{(8-11)}$. Similarly, the proportion of daily energy from sweetened beverages increased significantly from $11 \cdot 8 \%$ in 1965 to $21 \cdot 0 \%$ in 2002 in the $\mathrm{USA}^{(12)}$. Thus, health professionals recommend that children limit beverages high in energy such as regular soft drinks and fruit juices from their dietary intake ${ }^{(13,14)}$.

Theories and models of obesity risk ${ }^{(15-17)}$ suggest that the environment may play an important role in sweetened beverage consumption ${ }^{(18)}$. For example, the social environment, which encompasses the immediate physical surroundings, social relationships and cultural 
milieus within which defined groups of people function and interact ${ }^{(19)}$, may influence the consumption of soft drinks and juices. Within the home environment, parental characteristics, attitudes and behaviours are associated with the general eating behaviour of pre-school children ${ }^{(20-23)}$. For instance, having more than two children within the family, the mother having low education, the mother being unemployed or maternal perception that healthy food is less tasty were all associated with greater excess food intake (including sweet and savoury snacks, sweetened beverages and French fries) ${ }^{(24)}$. Conversely, the mother being 35 years of age or older and having a high health-attitude score have been associated with a lower excess food intake ${ }^{(24)}$. It is likely that parents and siblings may act as role models to encourage children to consume novel food and beverages ${ }^{(20,22,23)}$. A parent can control his/her child's sweetened beverage consumption by placing restrictions on the number of soft drinks permitted each day ${ }^{(25,26)}$, limiting the accessibility of regular soft drinks in the home and limiting participation in sedentary behaviours such as viewing television ${ }^{(27)}$. Evidence also suggests that imposing stringent parental controls can lead to preferences for energy-dense foods and drinks, such as sweetened beverages ${ }^{(20,21,23)}$.

Studies that have identified sociodemographic and behavioural risk factors for regular soft drink and sweetened beverage consumption among pre-school children are limited ${ }^{(24,25,28-30)}$. Increased sweetened beverage consumption was found to be associated with reduced milk intake among 3- to 7-year-old children ${ }^{(28)}$. Similarly, the enjoyment of drinking milk ${ }^{(25,29)}$ and water ${ }^{(25,31)}$ were associated with decreased likelihood of drinking regular soft drinks among children, while the desire to drink ${ }^{(30)}$ was associated with increased likelihood of regularly drinking soft drinks among pre-school children.

Beyond the household, neighbourhood environment may influence health behaviour and obesity risk among children $^{(32,33)}$. However, access to sweetened beverages in the neighbourhood has yet to be examined. A neighbourhood with limited access to grocery stores, and therefore limited access to healthy beverage choices, but having many fast-food restaurants and corner stores may encourage parents to purchase sweetened beverages. Therefore, more research is needed to determine if neighbourhoods and the accessibility of commercial establishments such as grocery stores, corner stores and fast-food restaurants influence sweetened beverage consumption among children.

Although several risk factors for consumption of sweetened beverages have been identified among schoolaged children and adolescents ${ }^{(9,10,25,26,31,34,35)}$, limited research has been conducted among pre-school children. Eating behaviours are developed at an early age and by adolescence these behaviours are well established ${ }^{(21)}$. Thus the determinants of eating behaviours will likely vary across these age groups ${ }^{(21)}$. For instance, pre-school children may have limited control in making decisions about dietary intake and are exposed to different environments compared with older children, such as the day-care setting ${ }^{(21)}$. Such dissimilarities may result in differing risk factors for regular soft drink consumption between these two groups of children. Therefore, the purpose of the present study was to determine the sociodemographic, behavioural and environmental characteristics associated with regular soft drink and fruit juice consumption among pre-school children.

\section{Methods}

Children who were attending a public health unit for preschool immunization in the Edmonton region of Alberta, Canada, between November 2005 and August 2007 were recruited for a longitudinal study on determinants of childhood obesity (Spatial Health Assessment of Preschooler's Environments (SHAPEs) $)^{(36,37)}$. The data reported herein are from the baseline phase of that study. Although not mandatory, a high proportion of children in the region visit these health centres for immunizations and other services from birth through to pre-school. Therefore, these health centres allow access to the majority of children in the region.

If contact was made with the parent, the recruitment rate was approximately $93 \%$. In total, 2114 parents participated with their 4- or 5-year-old child. Of these, 354 were excluded in the current analyses because they had at least one incomplete response to the socio-economic or demographic questions. The study was approved by the Health Research Ethics Board of the University of Alberta.

\section{Measures}

Parents were asked to complete a brief survey including questions on their child's food and beverage consumption, eating behaviours, physical activity and screen time. They were also asked some basic questions about their child's health and whether their child attended day care, a play school, pre-school or kindergarten. For the purpose of the present study, we focused on the portion of the questionnaire pertaining to beverage intake.

\section{Demographics}

The child's age and sex were collected by a questionnaire administered to their parents. Because children's addresses were not available, six-digit postal codes recorded in community health records were used to obtain neighbourhoodlevel information related to socio-economic status (SES). Specifically, the postal codes were geocoded (assigned a spatial reference) using the Postal Conversion File produced by Statistics Canada ${ }^{(38)}$ to locate the children's households within their dissemination areas. By using the dissemination areas, we were able to link Canada Census data to create SES variables. More specifically, based on the 
2006 census $^{(39)}$, a neighbourhood SES index was created for each dissemination area where the centroids of children's postal codes were located. This index was calculated by adding net educational level, median income of all census families, and then subtracting the proportion of unemployed ${ }^{(40)}$. Dissemination areas where the children resided were then classified into high, medium or low neighbourhood SES based upon a tertile split.

\section{Desire to drink and food fussiness}

The desire to drink (the quantity and frequency a child wants to drink) and food fussiness (pickiness) subscales of the Children's Eating Behaviour Questionnaire (CEBQ) were included as behavioural factors in the present study. The CEBQ is a thirty-five-item parent-report questionnaire designed to assess a range of eating behaviours of children ${ }^{(41)}$. Response options for all items were measured on a 5-item Likert scale ranging from 'never' to 'always'. The CEBQ has previously demonstrated good internal reliability, with Cronbach's $\alpha$ ranging between 0.72 and $0.91^{(41)}$, and good test-retest reliability with correlations ranging from $r=0.52$ to $r=0.87$ in pre-school children $^{(41)}$.

\section{Screen time}

Parents were asked to report their child's participation in sedentary behaviours, such as viewing television and playing video games. The American Academy of Pediatrics Committee on Nutrition ${ }^{(42)}$, the Canadian Paediatric Society ${ }^{(43)}$ and the Council on Sports Medicine and Fitness ${ }^{(44,45)}$ recommend no more than $2 \mathrm{~h}$ of nonschool-related screen time daily ${ }^{(45)}$. Therefore, screen time (television viewing and video game playing) was dichotomized into $\leq 2 \mathrm{~h} / \mathrm{d}$ and $>2 \mathrm{~h} / \mathrm{d}$.

\section{Beverage consumption}

An instrument developed by nutritionists in the public health authority was used to assess dietary intake of the children. Parents were asked, 'Thinking back over the past couple of weeks, how many servings has your child had of the following foods and beverages? Estimate the number of servings for each food or beverage, either over an average day or over an average week. If your child rarely or never has the food or beverage, write zero per day or per week'. The options for beverages included soft drinks, fruit juice, milk and water. Responses were dichotomized to zero regular soft drink consumption and any ( $>0$ cups) regular soft drink consumption in an average week. For fruit juice consumption, responses were categorized into $<1$ serving/d (where 1 serving $=1$ cup or $236 \mathrm{ml}$ ) and more than the recommended intake of fruit juice of $\geq 1$ serving $/ \mathrm{d}^{(13)}$. Milk consumption was dichotomized into $<2$ servings/d and $\geq 2$ servings/d (the recommendation for Milk and Alternatives from Eating Well with Canada's Food Guide). Water consumption was dichotomized into $<3$ servings $/ \mathrm{d}$ and $\geq 3$ servings $/ \mathrm{d}^{(13)}$.
Number of grocery stores, fast-food restaurants and convenience stores in the proximity of the child's residence

Information on location of commercial establishments in Edmonton for 2004 was supplied by the Health Inspection Division within Capital Health and/or found in the Alberta First Business Directory ${ }^{(46)}$. From these data sets, we selected grocery stores, fast-food outlets and convenience stores. Definitions for these establishments are given elsewhere ${ }^{(47)}$. ArcGIS version $9 \cdot 2$ (ESRI Inc., Redlands, CA, USA) was used to create aerial buffers of $1 \mathrm{~km}$ around the point indicating the location of a child's postal code. To calculate the number of establishments within each buffer, the Counts Points in Polygon analysis of the Hawth's tools extension for ArcGIS was utilized ${ }^{(48)}$. The number of grocery stores within $1 \mathrm{~km}$ was categorized into none, $1-3$ or $\geq 4$. The number of fastfood restaurants within $1 \mathrm{~km}$ was categorized into none, $1-10$ or $\geq 11$. The number of conveniences stores within $1 \mathrm{~km}$ was categorized into none, $1-3$ or $\geq 4$. Aerial buffers were employed instead of network buffers because, in comparison to older cities in North America, Edmonton is more of a modern city characterized by cul-de-sacs, informal alleys that may increase connectivity and road networks with many missing or incomplete sidewalks. As a result, aerial buffers might be a better way to capture the availability of stores within an individual's neighbourhood in comparison to a network buffer. An aerial buffer of $1 \mathrm{~km}$ around homes delimits a walkable neighbourhood ${ }^{(49)}$.

\section{Data analysis}

Descriptive statistics were conducted to determine the frequency distributions of the sociodemographic variables. To identify potential correlates of regular soft drink and fruit juice consumption, a step-up approach was adopted. First, $\chi^{2}$ tests were performed to test for differences in beverage consumption across sociodemographic strata. Second, in separate multivariate binomial regression models, the regression of regular soft drink and fruit juice consumption $v$. sociodemographic variables (sex, age, SES, attendance in day care) was preformed among the children who had no missing information ( $n$ 1760). Third, analyses were conducted adding the behavioural factors (milk consumption, water consumption, participation in screen time, desire to drink and fussiness to food). Sixty-four children were excluded due to missing response of one of the behavioural variables ( $n$ 1696). Finally, the environmental factors (grocery stores, fast-food restaurants and convenience stores within $1 \mathrm{~km}$ of the participant's residence) were added to the models. Due to missing data in the environmental variables, another 517 children were excluded ( $n$ 1179).

\section{Results}

Characteristics of the 1760 children whose parents responded to the survey appear in Table 1. Overall, the 
sample was evenly divided across age categories with slightly more boys than girls. Just over $55 \%$ of participants lived in high-SES neighbourhoods. Close to $90 \%$ of the children attended day care. Fewer than $45 \%$ of the

Table 1 Characteristics of pre-school students participating in the Spatial Health Assessment of Preschooler's Environments (SHAPEs) study, Edmonton, Alberta, Canada ( $n$ 1760)

\begin{tabular}{lrc}
\hline & $n$ & Proportion of study population (\%) \\
\hline Age & & \\
4 years & 892 & $50 \cdot 7$ \\
5 years & 868 & $49 \cdot 3$ \\
Sex & & \\
$\quad$ Boys & 906 & $51 \cdot 5$ \\
$\quad$ Girls & 854 & $48 \cdot 5$ \\
Neighbourhood SES & & \\
$\quad$ Low & 279 & $15 \cdot 9$ \\
$\quad$ Medium & 492 & $28 \cdot 0$ \\
$\quad$ High & 989 & $56 \cdot 2$ \\
Day care & & \\
$\quad$ No & 206 & $11 \cdot 7$ \\
Yes & 1554 & 88.3 \\
\hline
\end{tabular}

SES, socio-economic status. sample had parents report they participated in $>2 \mathrm{~h}$ of screen time daily. The average desire to drink and food fussiness scores were $2 \cdot 8(\mathrm{SD} 0 \cdot 9)$ and $3 \cdot 1$ (SD $0 \cdot 8$ ), respectively.

Of the sample, $68 \cdot 3 \%$ of the children consumed $\geq 2$ servings of milk daily while $36 \cdot 6 \%$ drank $\geq 3$ servings of water daily. In terms of sweetened beverage consumption, $46 \cdot 1 \%$ consumed regular soft drinks in the previous week and $29 \cdot 7 \%$ consumed $\geq 1$ serving of fruit juice daily.

Beverage consumption was associated with sociodemographic factors (see Table 2). Larger proportions of boys consumed regular soft drinks $\left(\chi^{2}=7 \cdot 99, P<0 \cdot 01\right)$ and milk $\left(\chi^{2}=7 \cdot 22, P<0 \cdot 01\right)$ in comparison to girls. Larger proportions of children living in low-SES neighbourhoods $\left(\chi^{2}=14 \cdot 14, P<0 \cdot 01\right)$ consumed regular soft drinks and fruit juices $\left(\chi^{2}=5 \cdot 85, P=0 \cdot 05\right)$ in comparison to those living in higher-SES neighbourhoods. Conversely, larger proportions of children from high-SES neighbourhoods consumed milk $\left(\chi^{2}=5 \cdot 83, P=0 \cdot 05\right)$. Children not attending day care were significantly more

Table 2 Bivariate analysis identifying correlates associated with regular soft drink consumption among pre-school children participating in the Spatial Health Assessment of Preschooler's Environments (SHAPEs) study, Edmonton, Alberta, Canada $(n$ 1760)

\begin{tabular}{|c|c|c|c|c|c|c|c|c|c|c|c|c|}
\hline & \multicolumn{3}{|c|}{$\begin{array}{l}\text { Regular soft drink at } \\
\text { least once weekly }\end{array}$} & \multicolumn{3}{|c|}{ Fruit juice $\geq 1$ serving/d } & \multicolumn{3}{|c|}{ Water $\geq 3$ servings/d } & \multicolumn{3}{|c|}{ Milk $\geq 2$ servings/d } \\
\hline & $n$ & $\%$ & $P$ & $n$ & $\%$ & $P$ & $n$ & $\%$ & $P$ & $n$ & $\%$ & $P$ \\
\hline Overall & 554 & $46 \cdot 1$ & & 357 & $29 \cdot 7$ & & 440 & $36 \cdot 6$ & & 822 & $68 \cdot 3$ & \\
\hline \multicolumn{13}{|l|}{ Sex } \\
\hline Boys & 305 & $49 \cdot 2$ & 0.02 & 175 & $28 \cdot 2$ & $0 \cdot 26$ & 226 & $36 \cdot 5$ & 0.93 & 442 & $71 \cdot 3$ & 0.02 \\
\hline Girls & 249 & $42 \cdot 7$ & & 182 & $31 \cdot 2$ & & 214 & $36 \cdot 7$ & & 380 & $65 \cdot 2$ & \\
\hline \multicolumn{13}{|l|}{ Age } \\
\hline 4-year-olds & 287 & $44 \cdot 6$ & 0.27 & 181 & $28 \cdot 1$ & $0 \cdot 20$ & 234 & $36 \cdot 3$ & $0 \cdot 85$ & 449 & $69 \cdot 7$ & 0.27 \\
\hline 5-year-olds & 267 & $47 \cdot 8$ & & 176 & $31 \cdot 5$ & & 206 & $36 \cdot 9$ & & 373 & $66 \cdot 7$ & \\
\hline \multicolumn{13}{|c|}{ Neighbourhood SES } \\
\hline Low & 127 & $54 \cdot 5$ & $<0.01$ & 87 & $37 \cdot 3$ & 0.02 & 90 & $38 \cdot 6$ & $0 \cdot 11$ & 148 & $63 \cdot 5$ & $0 \cdot 16$ \\
\hline Medium & 178 & $49 \cdot 6$ & & 103 & $28 \cdot 7$ & & 144 & $40 \cdot 1$ & & 244 & $68 \cdot 0$ & \\
\hline High & 249 & $40 \cdot 8$ & & 167 & $27 \cdot 3$ & & 342 & $32 \cdot 7$ & & 430 & $70 \cdot 4$ & \\
\hline \multicolumn{13}{|l|}{ Day care } \\
\hline No & 73 & $47 \cdot 1$ & $0 \cdot 78$ & 51 & $32 \cdot 9$ & $0 \cdot 35$ & 64 & $41 \cdot 3$ & $0 \cdot 19$ & 111 & $71 \cdot 6$ & 0.35 \\
\hline Yes & 481 & $45 \cdot 9$ & & 306 & $29 \cdot 2$ & & 376 & $35 \cdot 9$ & & 711 & $67 \cdot 8$ & \\
\hline \multicolumn{13}{|c|}{ Screen time status } \\
\hline Normal & 268 & $39 \cdot 5$ & $<0.01$ & 174 & $25 \cdot 6$ & $<0.01$ & 254 & $37 \cdot 4$ & $0 \cdot 50$ & 470 & $69 \cdot 2$ & 0.45 \\
\hline High & 286 & $54 \cdot 6$ & & 183 & $34 \cdot 9$ & & 186 & $35 \cdot 5$ & & 352 & $67 \cdot 2$ & \\
\hline \multicolumn{13}{|l|}{ Milk consumption } \\
\hline$<2$ servings/d & 182 & $47 \cdot 8$ & $0 \cdot 41$ & 107 & $28 \cdot 1$ & $0 \cdot 41$ & & & & & & \\
\hline$\geq 2$ servings/d & 372 & $45 \cdot 3$ & & 250 & $30 \cdot 4$ & & & & & & & \\
\hline \multicolumn{13}{|c|}{ Water consumption } \\
\hline$<3$ servings/d & 345 & $45 \cdot 2$ & 0.44 & 226 & $29 \cdot 6$ & 0.96 & & & & & & \\
\hline$\geq 3$ servings/d & 209 & $47 \cdot 5$ & & 131 & $29 \cdot 8$ & & & & & & & \\
\hline \multicolumn{13}{|c|}{ Grocery stores within $1 \mathrm{~km}$} \\
\hline None & 291 & $48 \cdot 0$ & 0.34 & 165 & $27 \cdot 2$ & $0 \cdot 14$ & 213 & $35 \cdot 1$ & 0.49 & 407 & $67 \cdot 2$ & 0.65 \\
\hline $1-3$ & 247 & $44 \cdot 3$ & & 181 & $32 \cdot 5$ & & 210 & $37 \cdot 7$ & & 388 & $69 \cdot 7$ & \\
\hline$\geq 4$ & 16 & $40 \cdot 0$ & & 11 & $27 \cdot 5$ & & 17 & $42 \cdot 5$ & & 27 & $67 \cdot 5$ & \\
\hline \multicolumn{13}{|c|}{ Fast-food restaurants within $1 \mathrm{~km}$} \\
\hline None & 178 & $44 \cdot 5$ & 0.35 & 117 & $29 \cdot 3$ & $0 \cdot 74$ & 152 & $38 \cdot 0$ & $0 \cdot 19$ & 269 & $67 \cdot 3$ & 0.78 \\
\hline $1-10$ & 300 & $45 \cdot 8$ & & 192 & $29 \cdot 3$ & & 226 & $34 \cdot 5$ & & 449 & $68 \cdot 5$ & \\
\hline$\geq 11$ & 76 & $51 \cdot 4$ & & 48 & $32 \cdot 4$ & & 62 & $41 \cdot 9$ & & 104 & $70 \cdot 3$ & \\
\hline \multicolumn{13}{|c|}{ Convenience stores within $1 \mathrm{~km}$} \\
\hline None & 147 & $41 \cdot 3$ & $<0.05$ & 106 & $29 \cdot 8$ & 0.96 & 124 & $34 \cdot 8$ & 0.56 & 246 & $69 \cdot 1$ & 0.90 \\
\hline $1-3$ & 348 & $47 \cdot 2$ & & 220 & $29 \cdot 8$ & & 272 & $36 \cdot 9$ & & 503 & $68 \cdot 2$ & \\
\hline$\geq 4$ & 59 & $54 \cdot 1$ & & 31 & $28 \cdot 4$ & & 44 & $40 \cdot 4$ & & 73 & $67 \cdot 0$ & \\
\hline
\end{tabular}

SES, socio-economic status. 
likely to drink water $\left(\chi^{2}=5 \cdot 07, P=0 \cdot 02\right)$ than those who attended day care. Regular soft drink consumption was not significantly associated with milk consumption $\left(\chi^{2}=0 \cdot 95, P=0 \cdot 33\right)$.

\section{Regular soft drink consumption}

At the first step, the multivariate logistic regression analysis (Table 3) indicated that girls (relative risk $(\mathrm{RR})=0.97,95 \%$ CI $0.78,0.96)$ were significantly less likely to have consumed regular soft drinks in comparison to boys. Children in low-SES $(\mathrm{RR}=1 \cdot 24,95 \% \mathrm{CI} 1 \cdot 09,2 \cdot 42)$ and mediumSES neighbourhoods $(\mathrm{RR}=1 \cdot 17,95 \% \mathrm{CI} 1 \cdot 04,1 \cdot 31)$ were significantly more likely to consume regular soft drinks in comparison to those in higher-SES neighbourhoods. When the behavioural covariates were included, similar results were obtained. However, 5-year-olds were now significantly more likely to consume regular soft drinks $(\mathrm{RR}=1 \cdot 10,95 \% \mathrm{CI} 1 \cdot 00,1 \cdot 22)$ in comparison to 4-yearolds. Children categorized as having a high screen time status $(\mathrm{RR}=1 \cdot 23,95 \% 1 \cdot 11,1 \cdot 36)$ or a higher desire to drink $(\mathrm{RR}=1 \cdot 15,95 \% \mathrm{CI} 1 \cdot 09,1 \cdot 21)$ were also significantly more likely to have consumed regular soft drinks. When the environmental factors were added, children who had $1-3$ grocery stores $(\mathrm{RR}=0 \cdot 84,95 \% \mathrm{CI}$ $0 \cdot 73,0.96)$ and $\geq 4$ grocery stores ( $R R=0.64,95 \% \mathrm{CI}$ $0 \cdot 42,0 \cdot 98)$ near their residence were significantly less likely to drink regular soft drinks. Having a fast-food restaurant or a convenience store within $1 \mathrm{~km}$ of the residence was not associated with regular soft drink consumption.

\section{Fruit juice consumption}

The multivariate regression analysis (Table 4) including only sociodemographic factors identified no significant risk factors for increased likelihood of fruit juice consumption. However, when behavioural factors were added, high participation in screen time $(\mathrm{RR}=1 \cdot 17,95 \%$ CI $1 \cdot 09,1 \cdot 25)$ and milk consumption ( $R R=1 \cdot 17,95 \% \mathrm{CI}$ $1 \cdot 07,1 \cdot 27$ ) were significantly associated with fruit juice consumption. When environmental factors were added to the model, high participation in screen time $(\mathrm{RR}=1 \cdot 16$, $95 \%$ CI $1 \cdot 06,1 \cdot 27)$ and milk consumption $(\mathrm{RR}=1 \cdot 17$,

Table 3 Multivariate analysis of correlates of regular soft drink consumption among pre-school children participating in the Spatial Health Assessment of Preschooler's Environments (SHAPEs) study, Edmonton, Alberta, Canada

\begin{tabular}{|c|c|c|c|c|c|c|}
\hline & \multicolumn{2}{|c|}{ Model 1 ( $n$ 1760) } & \multicolumn{2}{|c|}{ Model 2 ( $n$ 1696) } & \multicolumn{2}{|c|}{ Model 3 ( $n$ 1179) } \\
\hline & $\mathrm{RR}$ & $95 \% \mathrm{Cl}$ & $\mathrm{RR}$ & $95 \% \mathrm{Cl}$ & $\mathrm{RR}$ & $95 \% \mathrm{Cl}$ \\
\hline \multicolumn{7}{|l|}{ Sex } \\
\hline \multicolumn{7}{|l|}{ Boys (ref.) } \\
\hline Girls & $0 \cdot 97$ & $0.78,0.96$ & 0.90 & $0.81,0.99$ & 0.93 & $0.83,1.05$ \\
\hline \multicolumn{7}{|l|}{ Age } \\
\hline \multicolumn{7}{|l|}{ 4-year-olds (ref.) } \\
\hline 5-year-olds & 1.07 & $0.97,1.19$ & $1 \cdot 10$ & $1 \cdot 00,1 \cdot 22$ & $1 \cdot 08$ & $0 \cdot 96,1 \cdot 22$ \\
\hline \multicolumn{7}{|l|}{ Neighbourhood SES } \\
\hline \multicolumn{7}{|l|}{ High (ref.) } \\
\hline Medium & $1 \cdot 17$ & $1 \cdot 04,1 \cdot 31$ & $1 \cdot 12$ & $1 \cdot 00,1 \cdot 25$ & $1 \cdot 15$ & $0.99,1.34$ \\
\hline Low & $1 \cdot 24$ & $1 \cdot 09,2 \cdot 42$ & $1 \cdot 14$ & $1 \cdot 00,1 \cdot 31$ & $1 \cdot 17$ & $0.98,1.40$ \\
\hline \multicolumn{7}{|l|}{ Day care } \\
\hline \multicolumn{7}{|l|}{ No (ref.) } \\
\hline Yes & 0.98 & $0 \cdot 84,1 \cdot 14$ & $1 \cdot 01$ & $0 \cdot 87,1 \cdot 18$ & $1 \cdot 08$ & $0.91,1 \cdot 29$ \\
\hline \multicolumn{7}{|l|}{ Milk consumption } \\
\hline \multicolumn{7}{|l|}{$<2$ servings/d (ref.) } \\
\hline$\geq 2$ servings/d & & & 0.96 & $0 \cdot 87,1 \cdot 18$ & 0.94 & $0.83,1.07$ \\
\hline \multicolumn{7}{|l|}{ Water consumption } \\
\hline \multicolumn{7}{|l|}{$<3$ servings/d (ref.) } \\
\hline$\geq 3$ servings $/ d$ & & & $1 \cdot 01$ & $0.91,1 \cdot 12$ & $1 \cdot 00$ & $0 \cdot 89,1 \cdot 14$ \\
\hline \multicolumn{7}{|l|}{ Screen time status } \\
\hline \multicolumn{7}{|l|}{ Normal (ref.) } \\
\hline High & & & $1 \cdot 23$ & $1 \cdot 11,1 \cdot 36$ & $1 \cdot 28$ & $1 \cdot 13,1 \cdot 45$ \\
\hline Desire to drink & & & $1 \cdot 15$ & $1 \cdot 09,1 \cdot 21$ & $1 \cdot 15$ & $1.21,1.60$ \\
\hline Fussiness to food & & & 0.96 & $0.90,1.02$ & 1.00 & $0.93,1.07$ \\
\hline \multicolumn{7}{|c|}{ Grocery stores within $1 \mathrm{~km}$} \\
\hline \multicolumn{7}{|c|}{ None (ref.) } \\
\hline $1-3$ & & & & & $0 \cdot 84$ & $0.73,0.96$ \\
\hline$\geq 4$ & & & & & 0.64 & $0.42,0.98$ \\
\hline \multicolumn{7}{|c|}{ Fast-food restaurants within $1 \mathrm{~km}$} \\
\hline \multicolumn{7}{|c|}{ None (ref.) } \\
\hline $1-10$ & & & & & 0.97 & $0 \cdot 82,1 \cdot 14$ \\
\hline$\geq 11$ & & & & & $1 \cdot 20$ & $0.94,1.52$ \\
\hline \multicolumn{7}{|c|}{ Convenience stores within $1 \mathrm{~km}$} \\
\hline None (ref.) & & & & & & \\
\hline $1-3$ & & & & & $1 \cdot 11$ & $0.94,1.32$ \\
\hline$\geq 4$ & & & & & $1 \cdot 18$ & $0.91,1.52$ \\
\hline
\end{tabular}

$\mathrm{RR}$, relative risk; SES, socio-economic status; ref., reference category. 
Table 4 Multivariate analysis of correlates of fruit juice consumption among pre-school children participating in the Spatial Health Assessment of Preschooler's Environments (SHAPEs) study, Edmonton, Alberta, Canada

\begin{tabular}{|c|c|c|c|c|c|c|}
\hline & \multicolumn{2}{|c|}{ Model 1 ( $n$ 1760) } & \multicolumn{2}{|c|}{ Model 2 ( $n$ 1696) } & \multicolumn{2}{|c|}{ Model 3 (n 1179) } \\
\hline & RR & $95 \% \mathrm{Cl}$ & $\mathrm{RR}$ & $95 \% \mathrm{Cl}$ & $\mathrm{RR}$ & $95 \% \mathrm{Cl}$ \\
\hline \multicolumn{7}{|l|}{ Sex } \\
\hline \multicolumn{7}{|l|}{ Boys (ref.) } \\
\hline Girls & 0.99 & $0.93,1.06$ & $1 \cdot 01$ & $0.94,1.09$ & $1 \cdot 01$ & $0 \cdot 92,1 \cdot 10$ \\
\hline \multicolumn{7}{|l|}{ Age } \\
\hline 4-year-olds (ref.) & & & & & & \\
\hline 5-year-olds & $1 \cdot 01$ & $0.91,1 \cdot 38$ & $1 \cdot 03$ & $0 \cdot 96,1 \cdot 11$ & 1.06 & $0.97,1 \cdot 15$ \\
\hline \multicolumn{7}{|l|}{$\begin{array}{l}\text { Neighbourhood SES } \\
\text { High (ref.) }\end{array}$} \\
\hline $\begin{array}{l}\text { High (ref.) } \\
\text { Medium }\end{array}$ & 0.96 & $0.90,1.44$ & 0.95 & $0.87,1.03$ & 0.98 & $0.88,1.09$ \\
\hline Low & 1.04 & $0.94,1 \cdot 14$ & $1 \cdot 03$ & $0.93,1.13$ & 1.09 & $0.95,1.24$ \\
\hline \multicolumn{7}{|l|}{ Day care } \\
\hline No (ref.) & & & & & & \\
\hline Yes & 0.96 & $0 \cdot 86,1 \cdot 07$ & 0.96 & $0 \cdot 86,1 \cdot 07$ & $1 \cdot 02$ & $0 \cdot 89,1 \cdot 16$ \\
\hline \multicolumn{7}{|c|}{ Milk consumption } \\
\hline$\geq 2$ servings/d & & & $1 \cdot 17$ & $1 \cdot 07,1 \cdot 27$ & $1 \cdot 17$ & $1 \cdot 06,1 \cdot 29$ \\
\hline \multicolumn{7}{|c|}{ Water consumption } \\
\hline \multicolumn{7}{|l|}{ <3 servings/d (ref.) } \\
\hline$\geq 3$ servings/d & & & 0.91 & $0 \cdot 85,0.99$ & 0.93 & $0.85,1.02$ \\
\hline \multicolumn{7}{|l|}{ Screen time status } \\
\hline \multicolumn{7}{|l|}{ Normal (ref.) } \\
\hline High & & & $1 \cdot 17$ & $1 \cdot 09,1 \cdot 25$ & $1 \cdot 16$ & $1 \cdot 06,1 \cdot 27$ \\
\hline Desire to drink & & & $1 \cdot 03$ & $0.99,1.07$ & $1 \cdot 03$ & $0.98,1.08$ \\
\hline Fussiness to food & & & $1 \cdot 00$ & $0 \cdot 95,1 \cdot 04$ & 0.99 & $0.94,1.05$ \\
\hline \multicolumn{7}{|c|}{ Grocery stores within $1 \mathrm{~km}$} \\
\hline $1-3$ & & & & & 0.95 & $0 \cdot 86,1 \cdot 05$ \\
\hline$\geq 4$ & & & & & 0.96 & $0 \cdot 73,1 \cdot 26$ \\
\hline \multicolumn{7}{|c|}{ Fast-food restaurants within $1 \mathrm{~km}$} \\
\hline \multicolumn{7}{|c|}{ None (ref.) } \\
\hline $1-10$ & & & & & $1 \cdot 03$ & $0 \cdot 92,1 \cdot 16$ \\
\hline$\geq 11$ & & & & & $1 \cdot 00$ & $0 \cdot 83,1 \cdot 21$ \\
\hline \multicolumn{7}{|c|}{ Convenience stores within $1 \mathrm{~km}$} \\
\hline None (ref.) & & & & & & \\
\hline $1-3$ & & & & & $1 \cdot 02$ & $0 \cdot 91,1 \cdot 15$ \\
\hline$\geq 4$ & & & & & 0.90 & $0 \cdot 74,1 \cdot 11$ \\
\hline
\end{tabular}

$\mathrm{RR}$, relative risk; SES, socio-economic status; ref., reference category.

95\% CI 1.06, 1.29) were significantly associated with fruit juice consumption. No environmental factors were associated with fruit juice consumption.

\section{Discussion}

The purpose of the present study was to identify sociodemographic, behavioural and environmental correlates of sweetened beverages among pre-school children. Approximately $50 \%$ of the children consumed regular soft drinks and almost $30 \%$ drank more than 1 serving of fruit juice daily in the previous week. SES, participating in screen time for $>2 \mathrm{~h} / \mathrm{d}$, desire to drink and access to grocery stores within $1 \mathrm{~km}$ of the child's residence were significantly associated with regular soft drink consumption. Screen time participation, desire to drink and milk consumption were associated with fruit juice consumption. Although the population represented in the current investigation was pre-school children, similar trends in sweetened beverage consumption are found among older children in developed countries. Specifically, those from lower-SES backgrounds, who were boys and had high desire to drink were more likely to consume regular soft drinks ${ }^{(10,25,30,31)}$.

Although previous research has indicated regular soft drink consumption displaces milk consumption ${ }^{(29,50,51)}$, only milk consumption was associated with a greater likelihood of fruit juice consumption. This may be an indication that fruit juice and milk may both be perceived to be healthy by parents. Interventions may be needed in order to promote intake of beverages such as water as healthier options to fruit juice.

As observed in previous research with adolescents ${ }^{(52,53)}$, partaking in sedentary behaviour was associated with sweetened beverage consumption among our participants. This clustering of obesogenic behaviours is not uncommon among adolescents ${ }^{(54)}$ and may warrant more prevention efforts targeting young children. Furthermore, the finding that SES was no longer associated with fruit juice consumption after behavioural factors, such as screen time participation and desire to drink, were added to the model 
suggests the latter may mediate this relationship. Children from low-SES backgrounds are more likely to participate in high levels of screen time ${ }^{(55)}$. This may be due to parents from low-SES backgrounds being more likely to use the television as an inexpensive form of supervision ${ }^{(56,57)}$. Thus, implementing policies and programmes that make supervision of children more affordable and accessible, which can in turn lead to a reduction of sedentary behaviour participation, may be an effective strategy for reducing sweetened beverage consumption particularly among children from lower-SES backgrounds.

Taxation on regular soft drinks has been proposed as an effective strategy for decreasing sweetened beverage consumption ${ }^{(58-60)}$. Findings from a recent study indicate that state soft drink taxes in the USA have a small, yet significant impact on behaviour and weight ${ }^{(61)}$. However, children from poorer backgrounds are more likely to consume foods that are high in energy ${ }^{(62-64)}$ and more likely to consume regular soft drinks ${ }^{(65)}$. Thus, a tax on regular soft drinks may disproportionately affect families from lower-income backgrounds. These households have less disposable income and therefore a higher proportion of their income is spent on food in comparison to higherincome households. Any increase in the price of food and beverages would unfairly affect families already struggling to purchase food. However, other researchers argue there is no empirical evidence that a tax on regular soft drinks would be regressive and unfairly penalize lowincome individuals and households ${ }^{(66)}$. Nevertheless, our results indicate children from low-SES backgrounds are more likely to consume regular soft drinks and if taxation has no effect on the purchase of these beverages, excessive taxation of such products may have an economic impact on these families.

Another interesting finding from our investigation is that children who attended day care were significantly less likely to drink water. It is highly recommended that children should be encouraged to consume water to quench thirst ${ }^{(13)}$. The day-care setting would be ideal to promote water consumption and implement adoption of this behaviour since a large number of children are enrolled in such schools. Furthermore, tap water would be more affordable and accessible in comparison to other beverages such as fruit juice and milk.

Providing families with a variety of options to purchase groceries may also be beneficial in preventing excessive energy intake among pre-school children through regular soft drink consumption. Our study is one of the first to identify that accessible grocery stores within close proximity to place of residence is associated with a lower likelihood of regular soft drink consumption among children. This finding highlights the potential importance of the presence of commercial establishments that offer a variety of healthy food options within the neighbourhood. Other work indicates the presence of supermarkets in a neighbourhood is associated with reduced odds for obesity among residents ${ }^{(67)}$ and a high density of fastfood restaurants or convenience stores is associated with greater likelihood of being obese ${ }^{(47,68)}$. Therefore, the neighbourhood food environment may be an important determinant of sweetened beverage consumption among pre-school children.

Our study is not without several limitations that should be acknowledged. Many participants were excluded due to missing information, which restricts the comparability of the three models across the different sub-samples. The cross-sectional nature of the design limits our ability to establish cause-and-effect relationships. SES information was available only at the neighbourhood level, and not the individual level. Also, parental reports of eating behaviour and beverage intake may be subject to error because parents had limited opportunity to observe their child eating throughout the day, which is important to note since $88 \%$ of the children attended day care. Similarly, reporting of eating behaviours may have been biased due to the parent's motivation to provide socially desirable responses. Therefore, the strength of associations would most likely have been underestimated ${ }^{(69)}$. Finally, several correlates known to be associated with soft drink consumption were not included in the present study. For example, parental soft drink consumption and high access to regular soft drinks in the home ${ }^{(29)}$ have been found to be positively associated with soft drink consumption but were not included in the analyses.

The present investigation is one of the first to examine correlates of sweetened beverage consumption among pre-school children. Results indicate certain sociodemographic, behavioural and environmental factors are significantly related to consumption of these beverages. Future research should include longitudinal analyses to gain a better understanding of these factors and their influence on regular soft drink consumption among children as they age. Knowledge from such studies may aid public health practitioners to develop and implement interventions designed to prevent adoption of sweetened beverage consumption among children.

\section{Acknowledgements}

This work was supported by the Canadian Institutes of Health Research (CIHR), the Heart and Stroke Foundation of Canada, and the Social Sciences and Humanities Research Council (SSHRC). R.A.P. was supported by a CIHR-Training in Obesity Fellowship award. There are no conflicts of interest to declare. R.A.P. conceptualized the project, performed all analyses and wrote the article. J.C.S. conceptualized the project, is the principal investigator for the Spatial Health Assessment of Preschooler's Environments (SHAPEs) study, and contributed to the writing of the manuscript. N.C. is the health geographer of the SHAPEs study and helped conceptualize the geographical 
variables. L.C. helped developed the dietary intake assessment tools and contributed to the manuscript. K.S. helped with the nutrition aspects of the article and contributed to the manuscript.

\section{References}

1. Tremblay MS, Shields M, Laviolette M et al. (2010) Fitness of Canadian children and youth: results from the 2007-2009 Canadian Health Measures Survey. Health Rep 21, 7-20.

2. Poirier P, Giles TD, Bray GA et al. (2006) Obesity and cardiovascular disease: pathophysiology, evaluation, and effect of weight loss: an update of the 1997 American Heart Association Scientific Statement on Obesity and Heart Disease from the Obesity Committee of the Council on Nutrition, Physical Activity, and Metabolism. Circulation 113, 898-918.

3. Kaur H, Hyder ML \& Poston WS (2003) Childhood overweight: an expanding problem. Treat Endocrinol 2, 375-388.

4. James WP (2008) The epidemiology of obesity: the size of the problem. J Intern Med 263, 336-352.

5. Bray GA (2010) Soft drink consumption and obesity: it is all about fructose. Curr Opin Lipidol 21, 51-57.

6. Malik VS, Schulze MB \& Hu FB (2006) Intake of sugarsweetened beverages and weight gain: a systematic review. Am J Clin Nutr 84, 274-288.

7. Thundiyil JG, Christiano-Smith D, Greenberger $\mathrm{S}$ et al. (2010) Trimming the fat: identification of risk factors associated with obesity in a pediatric emergency department. Pediatr Emerg Care 26, 709-715.

8. French SA, Lin BH \& Guthrie JF (2003) National trends in soft drink consumption among children and adolescents age 6 to 17 years: prevalence, amounts, and sources, 1977/ 1978 to 1994/1998. J Am Diet Assoc 103, 1326-1331.

9. Rajeshwari R, Yang SJ, Nicklas TA et al. (2005) Secular trends in children's sweetened-beverage consumption (1973 to 1994): the Bogalusa Heart Study. J Am Diet Assoc 105, 208-214.

10. Naska A, Bountziouka V \& Trichopoulou A (2010) Soft drinks: time trends and correlates in twenty-four European countries. A cross-national study using the DAFNE (Data Food Networking) databank. Public Health Nutr 13, 1346-1355.

11. Nelson MC, Neumark-Sztainer D, Hannan PJ et al. (2009) Five-year longitudinal and secular shifts in adolescent beverage intake: findings from project EAT (Eating Among Teens)-II. J Am Diet Assoc 109, 308-312.

12. Duffey KJ \& Popkin BM (2007) Shifts in patterns and consumption of beverages between 1965 and 2002. Obesity (Silver Spring) 15, 2739-2747.

13. Alberta Health and Wellness, Population Health Strategies Branch, Public Health Division (2008) Alberta Nutrition Guidelines For Children and Youth: A Childcare, School and Recreation/Community Centre Resource Manual. Edmonton, AB: Government of Alberta.

14. Health Canada (2007) Eating Well with Canada's Food Guide. Ottawa: Minister of Public Works and Government Services Canada.

15. Davison KK \& Birch LL (2001) Childhood overweight: a contextual model and recommendations for future research. Obes Rev 2, 159-171.

16. Swinburn B, Egger G \& Raza F (1999) Dissecting obesogenic environments: the development and application of a framework for identifying and prioritizing environmental interventions for obesity. Prev Med 29, 563-570.

17. Swinburn B \& Egger G (2002) Preventive strategies against weight gain and obesity. Obes Rev 3, 289-301.
18. Galvez MP, Pearl M \& Yen IH (2010) Childhood obesity and the built environment. Curr Opin Pediatr 22, 202-207.

19. Barnett E \& Casper M (2001) A definition of 'social environment'. Am J Public Health 91, 465.

20. Campbell KJ, Crawford DA \& Hesketh KD (2007) Australian parents' views on their 5-6-year-old children's food choices. Health Promot Int 22, 11-18.

21. Birch LL \& Fisher JO (1998) Development of eating behaviors among children and adolescents. Pediatrics 101, 539-549.

22. Benton D (2004) Role of parents in the determination of the food preferences of children and the development of obesity. Int J Obes Relat Metab Disord 28, 858-869.

23. Savage JS, Fisher JO \& Birch LL (2007) Parental influence on eating behavior: conception to adolescence. J Law Med Ethics 35, 22-34.

24. Vereecken C \& Maes L (2010) Young children's dietary habits and associations with the mothers' nutritional knowledge and attitudes. Appetite 54, 44-51.

25. Grimm GC, Harnack L \& Story M (2004) Factors associated with soft drink consumption in school-aged children. $J \mathrm{Am}$ Diet Assoc 104, 1244-1249.

26. Nickelson J, Roseman MG \& Forthofer MS (2010) Associations between parental limits, school vending machine purchases, and soft drink consumption among Kentucky middle school students. J Nutr Educ Behav 42, 115-122.

27. Thomson M, Spence JC, Raine K et al. (2008) The association of television viewing with snacking behavior and body weight of young adults. Am J Health Promot 22, 329-335.

28. Keller KL, Kirzner J, Pietrobelli A et al. (2009) Increased sweetened beverage intake is associated with reduced milk and calcium intake in 3- to 7-year-old children at multi-item laboratory lunches. J Am Diet Assoc 109, 497-501.

29. Fiorito LM, Marini M, Mitchell DC et al. (2010) Girls' early sweetened carbonated beverage intake predicts different patterns of beverage and nutrient intake across childhood and adolescence. J Am Diet Assoc 110, 543-550.

30. Sweetman C, Wardle J \& Cooke L (2008) Soft drinks and 'desire to drink' in preschoolers. Int J Behav Nutr Phys Act 5, 60 .

31. Denney-Wilson E, Crawford D, Dobbins T et al. (2009) Influences on consumption of soft drinks and fast foods in adolescents. Asia Pac J Clin Nutr 18, 447-452.

32. Simen-Kapeu A, Kuhle S \& Veugelers PJ (2010) Geographic differences in childhood overweight, physical activity, nutrition and neighbourhood facilities: implications for prevention. Can J Public Health 101, 128-132.

33. Spence JC, Cutumisu N, Edwards J et al. (2008) Influence of neighbourhood design and access to facilities on overweight among preschool children. Int J Pediatr Obes 3, 109-116.

34. Bere E, Glomnes ES, te Velde SJ et al. (2008) Determinants of adolescents' soft drink consumption. Public Health Nutr 11, 49-56.

35. Striegel-Moore RH, Thompson D, Affenito SG et al. (2006) Correlates of beverage intake in adolescent girls: the National Heart, Lung, and Blood Institute Growth and Health Study. J Pediatr 148, 183-187.

36. Spence JC, Carson V, Casey L et al. (2011) Examining behavioural susceptibility to obesity among Canadian preschool children: the role of eating behaviours. Int J Pediatr Obes 6, e501-e507.

37. Carson V, Spence JC, Cutumisu N et al. (2010) Seasonal variation in physical activity among preschool children in a northern Canadian city. Res Q Exerc Sport 81, 392-399.

38. Statistics Canada (2008) Postal Code Conversion File (PCCFF), Reference Guide. March 2008 Postal codes. Statistics Canada Catalogue no. 92-153-G. Ottawa: Ministry of Industry. 
39. Statistics Canada (2008) 2006 Census of Population. Catalogue no. 94-581-XCB2006002. Ottawa, ON: Statistics Canada.

40. Demissie K, Hanley JA, Menzies D et al. (2000) Agreement in measuring socio-economic status: area-based versus individual measures. Chronic Dis Can 21, 1-7.

41. Wardle J, Guthrie CA, Sanderson S et al. (2001) Development of the children's eating behaviour questionnaire. J Child Psychol Psychiatry 42, 963-970.

42. Krebs NF \& Jacobson MS (2003) Prevention of pediatric overweight and obesity. Pediatrics 112, 424-430.

43. Canadian Paediatric Society (2003) Impact of media use on children and youth. Paediatr Child Health 8, 301-306.

44. Council on Sports Medicine and Fitness\& Council on School Health (2006) Active healthy living: prevention of childhood obesity through increased physical activity. Pediatrics 117, 1834-1842.

45. Tremblay MS, Leblanc AG, Janssen I et al. (2011) Canadian sedentary behaviour guidelines for children and youth. Appl Physiol Nutr Metab 36, 59-64; 65-71.

46. Alberta First Business Directory (2007) http://www.albertafirst. com/directory/ (accessed March 2007).

47. Spence JC, Cutumisu N, Edwards J et al. (2009) Relation between local food environments and obesity among adults. BMC Public Health 9, 192.

48. Beyer HL (2006) Hawth's Analysis Tools for ArcGIS, version 3.26. http://www.spatialecology.com (assessed June 2010).

49. Moudon A, Chanam L, Lee C et al. (2006) Operational definitions of walkable neighborhood: theoretical and empirical insights. J Phys Act Health 3, Suppl. 1, S99-S117.

50. Vartanian LR, Schwartz MB \& Brownell KD (2007) Effects of soft drink consumption on nutrition and health: a systematic review and meta-analysis. Am J Public Health 97, 667-675.

51. Marshall TA, Eichenberger Gilmore JM, Broffitt B et al. (2005) Diet quality in young children is influenced by beverage consumption. J Am Coll Nutr 24, 65-75.

52. de Bruijn GJ \& van den Putte B (2009) Adolescent soft drink consumption, television viewing and habit strength. Investigating clustering effects in the Theory of Planned Behaviour. Appetite 53, 66-75.

53. Kremers SP, van der Horst K \& Brug J (2007) Adolescent screen-viewing behaviour is associated with consumption of sugar-sweetened beverages: the role of habit strength and perceived parental norms. Appetite 48, 345-350.

54. Plotnikoff RC, Karunamuni N, Spence JC et al. (2009) Chronic disease-related lifestyle risk factors in a sample of Canadian adolescents. J Adolesc Health 44, 606-609.
55. Carson V, Spence JC, Cutumisu N et al. (2010) Association between neighborhood socioeconomic status and screen time among pre-school children: a cross-sectional study. BMC Public Health 10, 367.

56. Gordon-Larsen P, Griffiths P, Bentley ME et al. (2004) Barriers to physical activity: qualitative data on caregiver-daughter perceptions and practices. Am J Prev Med 27, 218-223.

57. Hanson MD \& Chen E (2007) Socioeconomic status, race, and body mass index: the mediating role of physical activity and sedentary behaviors during adolescence. J Pediatr Psychol 32, 250-259.

58. Sacks G, Veerman JL, Moodie M et al. (2011) 'Traffic-light' nutrition labelling and 'junk-food' tax: a modelled comparison of cost-effectiveness for obesity prevention. Int J Obes (Lond) 35, 1001-1009.

59. Bond ME, Williams MJ, Crammond B et al. (2010) Taxing junk food: applying the logic of the Henry tax review to food. Med J Aust 193, 472-473.

60. Rudd Center For Food Policy \& Obesity (2009) Soft Drink Taxes: A Policy Brief. New Haven,CT: Rudd Center For Food Policy \& Obesity, Yale University.

61. Fletcher JM, Frisvold D \& Tefft N (2010) Can soft drink taxes reduce population weight? Contemp Econ Policy 28, 23-35.

62. Drewnowski A (2003) Fat and sugar: an economic analysis. J Nutr 133, issue 3, 8385-840S.

63. Drewnowski A \& Specter SE (2004) Poverty and obesity: the role of energy density and energy costs. Am J Clin Nutr 79, 6-16.

64. Drewnowski A (2007) The real contribution of added sugars and fats to obesity. Epidemiol Rev 29, 160-171.

65. Janssen I, Katzmarzyk PT, Boyce WF et al. (2004) Overweight and obesity in Canadian adolescents and their associations with dietary habits and physical activity patterns. J Adolesc Health 35, 360-367.

66. Faulkner G, Grootendorst P, Nguyen V et al. (2010) Economic Policy, Obesity and Health: A Scoping Review. Final report submitted to the Heart and Stroke Foundation of Canada. Toronto, ON: Exercise Psychology Unit.

67. Bodor JN, Rice JC, Farley TA et al. (2010) The association between obesity and urban food environments. J Urban Health 87, 771-781.

68. Li F, Harmer P, Cardinal BJ et al. (2009) Obesity and the built environment: does the density of neighborhood fastfood outlets matter? Am J Health Promot 23, 203-209.

69. Rothman KJ, Greenland S \& Lash TL (2008) Modern Epidemiology, 3rd ed. Philadelphia, PA: Lippincott Williams \& Wilkins. 